\title{
Quantification of Tocopherols, Tocotrienols and $\gamma$-Oryzanol Contents of Local Rice Varieties in Northeastern Thailand
}

\author{
Grissana SUDTASARN ${ }^{1}$, Weerasak HOMSOMBAT ${ }^{2}$, \\ Somsong CHOTECHUEN ${ }^{3}$ and Varapong CHAMARERK ${ }^{3}$ \\ ${ }^{1}$ Ubon Ratchathani Rice Research Center, Ubon Ratchathani, Thailand \\ ${ }^{2}$ Nong Khai Rice Research Center, Nong Khai, Thailand \\ ${ }^{3}$ Bureau of Experts, Rice Deaprtment, Bangkok, Thailand
}

\begin{abstract}
Summary A total of 101 local rice varieties, composted of 85 glutinous and 16 nonglutinous varieties grown in wet season 2016, were analyzed for tocopherols (TOC), tocotrienols (T3) and $\gamma$-oryzanol (Orz). Two popular varieties, RD6 and KDML105, were used as standard checks for glutinous and non-glutinous varieties, respectively. $\gamma$-TOC was found in all glutinous varieties $(0.47-9.78 \mathrm{mg} / \mathrm{kg})$, which were higher than RD6 $(0.16 \mathrm{mg} / \mathrm{kg})$. $\alpha$-TOC was found in 40 varieties $(1.02-6.29 \mathrm{mg} / \mathrm{kg})$, only 6 varities were higher than RD6 $(3.95 \mathrm{mg} / \mathrm{kg}) . \delta$-T3 was found in 81 varieties $(0.57-7.00 \mathrm{mg} / \mathrm{kg})$, mostly varities were higher than RD6 $(0.67 \mathrm{mg} / \mathrm{kg}) . \gamma$-T3 was found in all glutinous varieties $(8.00-22.1 \mathrm{mg} /$ $\mathrm{kg}$ ), while RD6 contained $13.3 \mathrm{mg} / \mathrm{kg}$. For $\alpha$-T3, it was found in 39 glutinous varieties $(1.52-9.94 \mathrm{mg} / \mathrm{kg})$, mostly varities were higher than RD6 $(2.62 \mathrm{mg} / \mathrm{kg})$. Orz was found in all glutinous rice varieties $(276-638 \mathrm{mg} / \mathrm{kg}$ ), while RD6 contained $423 \mathrm{mg} / \mathrm{kg}$. $\gamma$-TOC was found in all non-glutinous varieties $(0.67-5.21 \mathrm{mg} / \mathrm{kg})$, which were higher than KDML105. $\alpha$-TOC was found in only 5 varieties $(1.92-2.83 \mathrm{mg} / \mathrm{kg})$, while KDML105 contained $2.23 \mathrm{mg} / \mathrm{kg}$. $\delta$-T3 was found in all non-glutinous varieties $(1.64-8.87 \mathrm{mg} / \mathrm{kg})$, which were higher than KDML105 $(0.71 \mathrm{mg} / \mathrm{kg}) \cdot \gamma$-T3 was also found in all non-glutinous varieties $(8.80-17.58 \mathrm{mg} / \mathrm{kg}$ ), while KDML105 contained $15.8 \mathrm{mg} / \mathrm{kg}$. $\alpha$-T3 was found in 9 nonglutinous varieties $(4.56-8.93 \mathrm{mg} / \mathrm{kg}$ ), while KDML105 contained only $0.86 \mathrm{mg} / \mathrm{kg}$. Orz was also found in all those non-glutinous varieties (272-469 mg/kg), while KDML105 contained $469 \mathrm{mg} / \mathrm{kg}$. These results indicate that $\gamma$-T3 was the highest vitamin E isomer present in all rice samples, while $\gamma$-TOC, $\alpha$-TOC, $\delta$-T3 and $\alpha$-T3 were present in trace amounts. Orz was found in all local rice varieties.
\end{abstract}

Key Words tocopherol, tocotrienol, gamma oryzanol, local rice, northeastern Thailand

Nowadays In Thailand, there are many famous local rice varieties which some farmers grow and for consuming and store their own seeds. The local rice is a major food and agricultural resource of the country especially on health concern and environmental conservation trend. The local rice varieties particularly on nutritional value become interesting. The previous research study on nutritional values of local rice varieties found that brown rice is a good source of many nutrients such as iron, zinc, copper, vitamin E and $\beta$-carotene (1). Nutritional values depending on the environmental condition, weather, fertilizer, soil, water, and rice genetics (1-2). Vitamin E exists in 8 forms $(\alpha-, \beta-, \gamma-$, and $\delta$-TOC and $\alpha-, \beta-, \gamma-$, and $\delta$-T3). Vitamin $\mathrm{E}$ inhibits oxidative processes in living tissues (3) and protects against liver oxidative damage (4). It shows antioxidants properties and will help strengthen the immune system and prevent many diseases; blood pressure, kidney disease, cancer and atherosclerosis. Orz is a natural compound with high nutrition and found only in rice, especially in rice bran. It has the same properties as vitamin $\mathrm{E}$ as an antioxidant and can prevent the oxidation of saturated fatty

E-mail: grissana.s@rice.mail.go.th acids better than tocopherol and tocotrienols. Brunswick Laboratories of the United States found that Orz can prevent oxidative of free radical with up to 6 times higher than vitamin E (5). The order of vitamin E, total TOC, total T3, and Orz contents was rice bran $>$ brown rice $>$ rice husk $>$ polished rice. $\gamma$-T3 was the highest vitamin $\mathrm{E}$ isomer present in all rice samples, while $\beta$-T, $\beta \mathrm{T} 3, \delta$-T, and $\delta$-T3 were present in trace amounts (6).

The research project on conservation and utilization of local rice varieties has been conducted with the aims of 1) developing rice varieties with good quality, meet farmers' preferences, and well adapt to local environments, and 2) supporting the utilization of local rice varieties in several aspects. An evaluation of nutritional values of local rice varieties was, therefore, conducted in order to obtain basic information for further utilization of those high nutritional value varieties, especially for health-concerned consumers..

\section{Materials and Methods}

Total of 101 local rice varieties, composted of 85 glutinous and 16 non-glutinous varieties grown in wet season 2016 at Sakon Nakorn Rice Research Center, were analyzed for TOC, T3 and Orz using reverse phase high 
Table 1. Tocopherols, tocotrienols and $\gamma$-oryzanol of 85 glutinous local rice varieties and RD6.

\begin{tabular}{|c|c|c|c|c|c|c|}
\hline \multirow{2}{*}{ Variety } & \multicolumn{2}{|c|}{ TOC $(\mathrm{mg} / \mathrm{kg})$} & \multicolumn{3}{|c|}{ T3 (mg/kg) } & \multirow{2}{*}{$\begin{array}{c}\text { Orz } \\
(\mathrm{mg} / \\
\mathrm{kg})\end{array}$} \\
\hline & $\gamma$ & $\alpha$ & $\delta$ & $\gamma$ & $\alpha$ & \\
\hline Dang Noi & 3.85 & 0.00 & 4.27 & 12.0 & 0.00 & 315 \\
\hline Daw Guem & 2.70 & 0.00 & 1.66 & 11.0 & 0.00 & 352 \\
\hline $\begin{array}{l}\text { Daw Hawm } \\
\text { (G.S. No. 4369) }\end{array}$ & 4.56 & 0.00 & 1.61 & 19.7 & 6.55 & 450 \\
\hline $\begin{array}{l}\text { Daw Hawm } \\
\text { (G.S. No. 1115) }\end{array}$ & 1.97 & 0.00 & 1.53 & 16.0 & 0.00 & 409 \\
\hline Dawk Hawm & 4.26 & 3.23 & 3.55 & 10.1 & 5.83 & 409 \\
\hline Dawk Kruem & 2.32 & 0.00 & 3.80 & 11.2 & 8.04 & 382 \\
\hline $\begin{array}{l}\text { Daw Noi } \\
\text { (G.S. No. 5609) }\end{array}$ & 2.82 & 0.00 & 4.22 & 12.6 & 0.00 & 373 \\
\hline $\begin{array}{l}\text { Daw Noi } \\
\text { (G.S. No. 5629) }\end{array}$ & 3.40 & 2.46 & 0.63 & 16.6 & 3.81 & 483 \\
\hline Daw Pla Siew & 3.67 & 2.34 & 0.57 & 47.4 & 4.18 & 373 \\
\hline Daw Pon & 3.98 & 0.00 & 0.00 & 11.7 & 0.00 & 500 \\
\hline Daw San Pa Tong & 2.93 & 1.63 & 3.17 & 10.9 & 0.00 & 385 \\
\hline Daw Tia & 2.26 & 0.00 & 0.93 & 11.1 & 7.99 & 433 \\
\hline Daw Yuan & 3.91 & 3.21 & 1.60 & 20.4 & 5.33 & 501 \\
\hline E-Pon & 3.57 & 2.38 & 4.47 & 16.9 & 0.00 & 434 \\
\hline E-Dam Dang & 2.84 & 0.00 & 4.09 & 11.6 & 4.84 & 402 \\
\hline E-Leaung & 2.53 & 0.00 & 1.13 & 12.9 & 0.00 & 401 \\
\hline E-Khao Glang & 3.48 & 0.00 & 0.77 & 9.8 & 0.00 & 614 \\
\hline E-Nawn Nawi & 2.38 & 0.00 & 0.90 & 13.2 & 0.00 & 471 \\
\hline E-Tang & 2.46 & 0.00 & 0.65 & 12.8 & 0.00 & 483 \\
\hline Gra Doog Ngoo & 3.85 & 0.00 & 2.80 & 14.1 & 5.41 & 367 \\
\hline Hang Yee & 4.17 & 0.00 & 3.88 & 11.5 & 5.80 & 319 \\
\hline $\begin{array}{l}\text { Hawm } \\
\text { (G.S. No. 245151) }\end{array}$ & 4.22 & 3.06 & 1.35 & 18.8 & 5.54 & 315 \\
\hline $\begin{array}{l}\text { Hawm } \\
\text { (G.S. No. 4433) }\end{array}$ & 3.61 & 4.57 & 1.32 & 22.1 & 7.91 & 421 \\
\hline Hawm Dang & 4.33 & 3.11 & 2.15 & 15.3 & 6.69 & 434 \\
\hline Hawm Daw & 3.14 & 0.00 & 3.54 & 12.8 & 0.00 & 412 \\
\hline Hawm Meaung Lei & 2.05 & 3.35 & 1.58 & 13.4 & 4.40 & 310 \\
\hline Hawm Nang Nuan & 3.08 & 2.22 & 0.79 & 15.3 & 6.36 & 343 \\
\hline Hawm Pae Palo & 2.79 & 0.00 & 1.46 & 12.9 & 5.48 & 290 \\
\hline $\begin{array}{l}\text { Hawm Pama } \\
\text { (G.S. No. 3210) }\end{array}$ & 2.10 & 2.63 & 1.59 & 13.7 & 0.00 & 342 \\
\hline $\begin{array}{l}\text { Hawm Pama } \\
\text { (G.S. No. 13739) }\end{array}$ & 1.61 & 0.00 & 4.36 & 13.9 & 4.87 & 383 \\
\hline Hawm Tung & 2.53 & 2.43 & 1.51 & 15.3 & 4.61 & 360 \\
\hline Hawm Sahngeam & 0.48 & 4.32 & 2.48 & 14.8 & 7.42 & 315 \\
\hline Hawm Udom & 1.73 & 2.77 & 1.72 & 14.1 & 4.59 & 350 \\
\hline Kaset & 4.85 & 2.89 & 2.44 & 14.2 & 0.00 & 424 \\
\hline Kaset Daw & 3.06 & 0.00 & 2.00 & 17.0 & 5.52 & 277 \\
\hline Khao Daw & 2.23 & 0.00 & 0.68 & 10.3 & 0.00 & 479 \\
\hline $\begin{array}{l}\text { Khao' Gam } \\
\text { (G.S. No. 23171) }\end{array}$ & 5.19 & 2.92 & 2.87 & 19.5 & 1.52 & 439 \\
\hline $\begin{array}{l}\text { Khao' Gam } \\
\text { (G.S. No. 23175) }\end{array}$ & 2.26 & 1.83 & 5.28 & 12.2 & 0.00 & 536 \\
\hline $\begin{array}{l}\text { Khao' Hao } \\
\text { (G.S. No. 23172) }\end{array}$ & 4.51 & 2.95 & 1.79 & 12.6 & 0.00 & 499 \\
\hline $\begin{array}{l}\text { Khao' Hao } \\
\text { (G.S. No. 23173) }\end{array}$ & 1.11 & 0.00 & 2.63 & 9.1 & 0.00 & 408 \\
\hline $\begin{array}{l}\text { Khao' Hao } \\
\text { (G.S. No. 23174) }\end{array}$ & 2.73 & 0.00 & 4.41 & 8.0 & .00 & 505 \\
\hline Khao’ Hawm & 9.78 & 3.81 & 1.30 & 15.3 & 5.51 & 297 \\
\hline
\end{tabular}

Table 1. (continued)

\begin{tabular}{|c|c|c|c|c|c|c|}
\hline \multirow{2}{*}{ Variety } & \multicolumn{2}{|c|}{ TOC $(\mathrm{mg} / \mathrm{kg})$} & \multicolumn{3}{|c|}{ T3 (mg/kg) } & \multirow{2}{*}{$\begin{array}{c}\text { Orz } \\
(\mathrm{mg} / \\
\mathrm{kg})\end{array}$} \\
\hline & $\gamma$ & $\alpha$ & $\delta$ & $\gamma$ & $\alpha$ & \\
\hline Khao' Hawm Daw & 2.73 & 4.98 & 1.29 & 18.4 & 0.00 & 325 \\
\hline Khao Khiew Kway & 2.23 & 0.00 & 4.57 & 12.7 & 0.00 & 276 \\
\hline $\begin{array}{l}\text { Khao Noi } \\
\text { (G.S. No. 5643) }\end{array}$ & 2.70 & 0.00 & 4.89 & 12.3 & 0.00 & 506 \\
\hline $\begin{array}{l}\text { Khao Noi } \\
\text { (G.S. No. 85004) }\end{array}$ & 3.61 & 1.42 & 4.31 & 13.1 & 3.82 & 320 \\
\hline Khao Yai & 3.77 & 0.00 & 0.00 & 10.6 & 0.00 & 638 \\
\hline Leuang Boonma & 3.17 & 0.00 & 3.42 & 11.9 & 0.00 & 455 \\
\hline Leuang Gaew & 2.76 & 0.00 & 4.28 & 11.9 & 6.88 & 429 \\
\hline Ma Nam & 2.84 & 1.91 & 1.70 & 13.3 & 0.00 & 294 \\
\hline Ma Phai & 2.23 & 3.95 & 4.29 & 12.7 & 9.94 & 407 \\
\hline Ma Prang & 2.64 & 0.00 & 0.88 & 14.1 & 0.00 & 489 \\
\hline Mhak Yom & 5.29 & 1.02 & 2.05 & 16.4 & 0.00 & 382 \\
\hline Muay Hin & 3.26 & 3.20 & 2.16 & 13.6 & 0.00 & 302 \\
\hline $\begin{array}{l}\text { Nahng Boon Ma } \\
\text { (G.S. No. 23217) }\end{array}$ & 3.18 & 3.49 & 1.84 & 21.6 & 0.00 & 442 \\
\hline $\begin{array}{l}\text { Nahng Boon Ma } \\
\text { (G.S. No. } 87087 \text { ) }\end{array}$ & 2.74 & 0.00 & 3.29 & 12.3 & 0.00 & 542 \\
\hline Nahng Nee & 4.56 & 0.00 & 1.71 & 13.6 & 5.40 & 281 \\
\hline Nahng Sa-ad & 4.13 & 0.00 & 0.00 & 12.8 & 0.00 & 435 \\
\hline Nahng Suan & 2.66 & 0.00 & 4.10 & 13.3 & 0.00 & 419 \\
\hline Niaw Dam & 4.02 & 3.06 & 1.38 & 11.6 & 0.00 & 597 \\
\hline Niaw Dang & 4.25 & 0.00 & 2.03 & 13.7 & 0.00 & 534 \\
\hline Niaw E-Noi & 3.20 & 0.00 & 0.87 & 9.6 & 0.00 & 501 \\
\hline Niaw Hawm & 2.83 & 2.41 & 1.88 & 14.1 & 0.00 & 398 \\
\hline Niaw Kam Noi & 4.26 & 0.00 & 2.90 & 15.6 & 6.75 & 357 \\
\hline Niaw Khe’ Kway & 3.10 & 0.00 & 0.79 & 9.9 & 0.00 & 516 \\
\hline Niaw Khiaw Ngoo & 3.63 & 2.98 & & 0 & 0 & 327 \\
\hline Niaw Mali & 0.72 & 4.68 & 1.66 & 19.4 & 6.69 & 464 \\
\hline Niaw Som Hong & 3.39 & 0.00 & 0.00 & 10.4 & 0.00 & 604 \\
\hline Noi Boon Ma & 3.28 & 0.00 & 4.12 & 13.1 & 6.08 & 488 \\
\hline Pama Hawm & 1.99 & 2.12 & 1.76 & 21.6 & 0.00 & 421 \\
\hline Pan Poo & 2.84 & 0.00 & 4.52 & 12.7 & 7.03 & 411 \\
\hline Ped Nam & 2.94 & 5.11 & 3.71 & 15.0 & 5.53 & 405 \\
\hline Plawng Aew & 3.01 & 2.74 & 1.01 & 13.7 & 5.83 & 357 \\
\hline Plong Aew & 3.63 & 2.00 & 2.32 & 11.6 & 6.23 & 403 \\
\hline Pra In & 2.59 & 2.06 & 2.14 & 17.4 & 6.19 & 412 \\
\hline Pram & 3.26 & 3.06 & 1.32 & 18.4 & 0.00 & 476 \\
\hline Rak Pai & & 0.00 & 5.00 & 11.1 & 0.00 & 502 \\
\hline Sa-Nai & 1.79 & 0.00 & 7.00 & 12.4 & 5.31 & 363 \\
\hline Sam Deaun Hang & 4.42 & 3.41 & 1.66 & 14.5 & 0.00 & 407 \\
\hline Sam Ruang & 3.91 & 2.08 & 2.79 & 14.0 & 6.16 & 324 \\
\hline San Pa Tong Dan & 3.41 & 0.00 & 2.19 & 14.1 & 0.00 & 428 \\
\hline Ta Plin & 3.09 & 0.00 & 2.26 & 13.6 & 0.00 & 450 \\
\hline Ta Prod Lian & 3.51 & 0.00 & 0.77 & 10.6 & 0.00 & 371 \\
\hline Tan Gon & 0.47 & 6.29 & 2.12 & 13.5 & 8.26 & 457 \\
\hline RD6 (ck) & 0.16 & 3.95 & 0.67 & 13.3 & 2.62 & 423 \\
\hline
\end{tabular}

G.S. No. $=$ Genetic Stock Number. 
Table 2. Tocopherols, tocotrienols and $\gamma$-oryzanol of 16 non-glutinous local rice varieties and KDML 10.

\begin{tabular}{lcccccc}
\hline \multirow{1}{*}{\multicolumn{1}{c}{ Variety }} & TOC $(\mathrm{mg} / \mathrm{kg})$ & \multicolumn{2}{c}{ T3 } & $(\mathrm{mg} / \mathrm{kg})$ & $\begin{array}{c}\text { Orz } \\
(\mathrm{mg} /\end{array}$ \\
\cline { 2 - 7 } & $\gamma$ & $\alpha$ & $\delta$ & $\gamma$ & $\alpha$ & $\mathrm{kg})$ \\
\hline Choo Chat & 2.49 & 0.00 & 4.14 & 15.5 & 7.35 & 272 \\
Daw Dang & 3.91 & 0.00 & 6.18 & 13.3 & 0.00 & 302 \\
Daw Khao & 1.92 & 2.61 & 4.55 & 14.2 & 5.27 & 436 \\
Hawm Jan & 2.28 & 0.00 & 1.84 & 14.8 & 0.00 & 349 \\
Jao Dang & & & & & & \\
(G.S. No. 5672) & 5.21 & 0.00 & 2.83 & 17.6 & 8.35 & 376 \\
Jao Dang & 4.07 & 0.00 & 8.87 & 15.5 & 0.00 & 426 \\
(G.S. No. 14511) & & & & & & \\
Jao Dang & 2.63 & 0.00 & 6.63 & 11.5 & 6.19 & 353 \\
(G.S. No. 13735) & & & & & & \\
Khan Nahng & 2.20 & 2.60 & 2.90 & 15.7 & 0.00 & 367 \\
Khao & 2.61 & 2.83 & 2.79 & 13.5 & 6.58 & 441 \\
Khao Gaw Diaw & 1.78 & 0.00 & 2.56 & 8.8 & 6.29 & 307 \\
Khao' Jao Leuang 11 & 2.03 & 0.00 & 6.61 & 11.6 & 0.00 & 324 \\
Kra Dab Tawng & 0.67 & 2.63 & 2.40 & 13.5 & 8.93 & 292 \\
Nahng Gang & 2.34 & 0.00 & 2.76 & 14.5 & 4.56 & 469 \\
Pong Aew & 5.10 & 1.92 & 2.17 & 15.7 & 6.74 & 392 \\
Taw Chaw Daw & 1.33 & 0.00 & 4.48 & 10.0 & 0.00 & 361 \\
Sam Ruang & 2.14 & 0.00 & 1.64 & 12.4 & 0.00 & 370 \\
KDML 105 (ck) & 0.52 & 2.23 & 0.71 & 15.8 & 0.86 & 469 \\
\hline & & & & & & \\
\hline
\end{tabular}

G.S. No. $=$ Genetic Stock Number.

performance liquid chromatography (RP-HPLC) at Ubon Ratchatani Rice Research Center. Two popular varieties, RD6 and KDML105, were used as standard checks for glutinous and non-glutinous varieties, respectively.

\section{Results}

The results showed that total of Vitamin E contents of different varieties of brown rice ranged between 0.47 and $47.4 \mathrm{mg} / \mathrm{kg}$. TOC were mostly found in the form of either $\gamma$ - or $\alpha$-TOC, while T3 were found in the forms of $\delta$-, $\gamma$ - and $\alpha$-T3. Among glutinous varieties, $\gamma$-TOC was found in all 85 glutinous varieties with the contents ranging from $0.47-9.78 \mathrm{mg} / \mathrm{kg}$, which were higher than that of the standard check, RD6 $(0.16 \mathrm{mg} / \mathrm{kg})$. For $\alpha$-TOC, it was found in 40 varieties with the contents ranging from $1.02-6.29 \mathrm{mg} / \mathrm{kg}$, while RD6 contained $3.95 \mathrm{mg} / \mathrm{kg}$. For T3, $\delta$-T3 was found in 81 varieties with the contents ranging from $0.57-7.00 \mathrm{mg} / \mathrm{kg}$, while the standard check (RD6) contained $0.67 \mathrm{mg} / \mathrm{kg} . \gamma$-T3 was found in all glutinous varieties being tested with the contents ranging from $8.00-22.1 \mathrm{mg} / \mathrm{kg}$, while RD6 contained $13.3 \mathrm{mg} / \mathrm{kg}$. For $\alpha$-T3, it was found in $39 \mathrm{glu}-$ tinous varieties with the contents ranging from 1.52$9.94 \mathrm{mg} / \mathrm{kg}$, while RD6 contained $2.62 \mathrm{mg} / \mathrm{kg}$. For Orz, it was found in all glutinous rice varieties being tested with the contents ranging from $276-638 \mathrm{mg} / \mathrm{kg}$, while RD6 contained $423 \mathrm{mg} / \mathrm{kg}$ (Table 1).

Among 16 non-glutinous varieties being tested, it was found that all those varieties contained $\gamma$-TOC with the contents ranging from $0.67-5.21 \mathrm{mg} / \mathrm{kg}$, which were higher than that of the standard check , KDML $105(0.52 \mathrm{mg} / \mathrm{kg})$. For $\alpha$-TOC, it was found in only 5 varieties being tested with the contents ranging from $1.92-2.83 \mathrm{mg} / \mathrm{kg}$, while KDML 105 contained $2.23 \mathrm{mg} / \mathrm{kg}$. For T3, $\delta$-T3 was found in all varieties with the contents ranging from $1.64-8.87 \mathrm{mg} / \mathrm{kg}$, which were higher than that of the standard check, KDML 105 $(0.71 \mathrm{mg} / \mathrm{kg}) . \gamma-\mathrm{T} 3$ was also found in all varieties being tested with the contents ranging from $8.80-17.58 \mathrm{mg} /$ $\mathrm{kg}$, while KDML 105 contained $15.8 \mathrm{mg} / \mathrm{kg}$. For $\alpha$-T3, it was found in 9 non-glutinous varieties being tested with the contents ranging from $4.56-8.93 \mathrm{mg} / \mathrm{kg}$, which were higher than KDML 105, contained only $0.86 \mathrm{mg} /$ $\mathrm{kg}$. For Orz, it was found in all 16 non-glutinous varieties being tested with the contents ranging from 272 $469 \mathrm{mg} / \mathrm{kg}$, while KDML 105 contained $469 \mathrm{mg} / \mathrm{kg}$ (Table 2).

\section{Discussion}

$\gamma$-TOC and $\gamma$-T3 were found in all glutinous and nonglutinious local rice varieties, while $\alpha$-TOC and $\alpha$-T3 were found in some local rice varieties. Most local rice varieties contain higher $\gamma$-TOC, $\delta$-T3 and $\alpha$-T3 than standard check varieties (RD6 and KDML 105). $\gamma$-T3 was the highest vitamin $\mathrm{E}$ isomer present in all rice samples, while $\gamma$-TOC, $\alpha$-TOC, $\delta$-T3 and $\alpha$-T3 were present in trace amounts. As same as Huang SH and Ng LT (2011), which found that $\gamma$-T3 was the highest vitamin $\mathrm{E}$ isomer present in all 16 commercial rice varieties in Taiwan (6). In japonica rice, $\alpha$-TOC, $\alpha$-T3, and $\gamma$-T3 were the most abundant homologs, while in indica rice the most abundant were $\gamma$-T3, $\alpha$-TOC, and $\alpha$-T3 (7). Content of vitamin $\mathrm{E}$ isomers varied among local rice varieties both within and between grain color groups. However, no obvious difference in TOC, T3 and $\gamma$-oryzanol content was noted between black- and red-colored local rice varieties, while Shammugasamy et al. found that black-pigmented rice had higher contents of TOC and T3 than non-pigmented rice and red-pigmented rice in 58 different varieties of whole rice cultivated in Malaysia (8).

In conclusion, this study provides information on vitamin E and Orz contents of different local rice varieties that would be beneficial for decision making in genetic breeding of bioactive compound-rich rice varieties and for further utilization of those high nutritional value varieties, especially for health-concerned consumers.

\section{Disclosure of State of COI}

No conflicts of interest to be declared.

\section{Acknowledgments}

We are grateful to Rice Department whose support fund for participation to The 3rd International Symposium on Rice Science in Global Health for this paper possible.

\section{REFERENCES}

1) Kongkachuichai R, Charoensiri R, Kringkasemsri A, Vanavichit A, Ruangphayak S. 2008. Iron zinc copper 
vitamin $\mathrm{E}$ beta-carotene and lutein contents in various land-race rice varieties in Thailand. Journal of the National Research Council of Thailand. Nat Sci 40(2): 13-32.

2) Bergman C J, Xu Z. 2003. Genotype and environment effects on tocopherol, tocotrienol and $\gamma$-oryzanol contents of southern U.S. rice. Cereal Chem 80(4): 446-449.

3) Fukui K, Nakamura K, Shirai M, Hirano A, Takatsu H, Urano S. 2015. Long-term vitamin E-deficient mice exhibit cognitive dysfunction via elevation of brain oxidation. J Nutr Sci Vitaminol 61(5): 362-368.

4) Ohta Y, Yashiro K, Ohashi K, Horikoshi Y, Kusumoto C, Matsura T, Fukuzawa K. 2015. Effect of dietary vitamin E supplementation on liver oxidative damage in rats with water-immersion restraint stress. J Nutr Sci Vitaminol 61(2): 113-122.
5) Rachada. Local rice, The good that is almost forgotten. 2011 [cited 2014 Sep 10] Available from: http://www. oknation.net/blog/rachada/2011/11/13/entry-7.

6) Huang SH, Ng LT. 2011. Quantification of tocopherols, tocotrienols, and $\gamma$-oryzanol contents and their distribution in some commercial rice varieties in Taiwan. J Agric Food Chem 59: 11150-11159.

7) Riana J. B. Heinemann, Zhimin Xu, J. Samuel Godber and Ursula M. Lanfer-Marquez. 2008. Tocopherols, tocotrienols, and $\gamma$-oryzanol contents in japonica and indica subspecies of rice (Oryza sativa L.) cultivated in Brazil. Cereal Chem 85(2): 243-247.

8) Shammugasamy, B., Y. Ramakrishnan, H.M. Ghazali and K. Muhammad. 2015. Tocopherol and tocotrienol contents of different varieties of rice in Malaysia. J Sci Food Agric 95(4): 672-678. 